\title{
PERFORMANCE ANALYSIS OF PRIORITIZED TOKEN RING
}

\author{
Kijoon Chae \\ and \\ Arne A. Nilsson
}

Center for Communications and Signal Processing Department of Electrical and Computer Engineering North Carolina State University

April 1989

CCSP-TR-89/8 


\section{ABSTRACT}

An analytic model for prioritized token ring is presented in this report. Its protocol is based on prioritized token ring with reservation (R-PTR). Since the protocol of the RPTR is simple and the performance of the R-PTR is not inferior to that of the IEEE-PTR under almost all traffic load environments, we use the R-PTR as our token ring model. By using the properties of Markovian process, the expressions for average throughput and average packet transmission delay are derived. The results obtained from the analytic model are compared with that of the simulation model. 


\section{INTRODUCTION}

In this report, we present an analytic model for performance evaluation of token ring with priority scheme. Its protocol is based on prioritized token ring with reservation (R-PTR) [1] which is similar to IEEE standard 802.5 [2].

In token ring, a token circulates around the ring when all stations are idle. A station wishing to transmit must wait until it detects a free token passing by. If it seizes a free token, it changes the free token to a busy token and transmits a packet which is appended to the end of the busy token. When the station completes the data transmission, it purges its transmitted packet and changes the busy token to a free token.

In the R-PTR, the priority operation is accomplished by assigning a priority to the free token. During one-round circulation, a busy token collects information about the highest priority of all access waiting packets at all stations. When a new free token is generated, its priority is given by the highest level of priority among the packets waiting to be transmitted.

The main differences between the R-PTR studied in this report and the IEEE-PTR are as follows.

First, in the IEEE-PTR, the priority of a new free token is given by the maximum of the current token priority and the highest priority of access waiting packets known to the busy token during its one-round circulation. In the R-PTR, the priority is given by the highest priority of the access waiting packets.

Second, in the IEEE-PTR, two types of stacks, $S_{r}$ and $S_{x}$, are used to store old and new values of token priority, respectively. Such stacks are not used in the R-PTR. Since 
each station maintains its own copy of those stacks in IEEE-PTR, the space for the stacks becomes large in the situation of very large number of stations. In addition, the manipulation of the stacks makes the IEEE-PTR rather complex.

Shen, Masuyama, Muro and Hasegawa [1] prove that the performance of the R-PTR is not inferior to that of the IEEE-PTR under almost all traffic load environments. Because of the above reasons, we use the R-PTR as our token ring model.

\section{MODEL DESCRIPTIONS}

Our model is shown in Figure 1 and can be characterized by the following assumptions:

(1) The number of stations is $N$ and the priority level of a packet is a uniformly distributed random integer between 0 and 7 . Level 0 is the lowest priority and level 7 is the highest priority.

(2) Each station has only one buffer whose size is one.

(3) "Round-robin" packet transmission strategy is considered, i.e., a station can transmit only one packet each time it captures a free token.

(4) The state of the token ring is represented by $\left(n_{0}, \cdots, n_{7}, f\right)$, where $n_{i}$ is the number of stations with a level $i$ packet and $f$ is the priority level of a free token. The sum of all $n_{i}{ }^{\prime} s$ must be less than or equal to $N$.

(5) A stream of packets arrives at each station according to Poisson process with mean value $\lambda$. 
(6) The channel times are normalized by the transmission time of a packet, i.e., the size of a packet is 1 unit time.

(7) The propagation delay between the nearest two stations is $r$ unit time.

In Figure 1, a free token arrives at station $\mathrm{A}$, and then station $\mathrm{B}$. We consider the following four time instants:

$t A$ : the instant that station A seizes a free token

$t A^{\prime}:$ the instant that station $\mathrm{A}$ issues a free token

$t B$ : the instant that station B seizes the free token issued by station A

$t B^{\prime}:$ the instant that station $B$ issues a free token.

Each time instant is a Markov renewal point since the state at any instant depends on both the state at the previous instant and the number of arrivals between the current and previous instants.

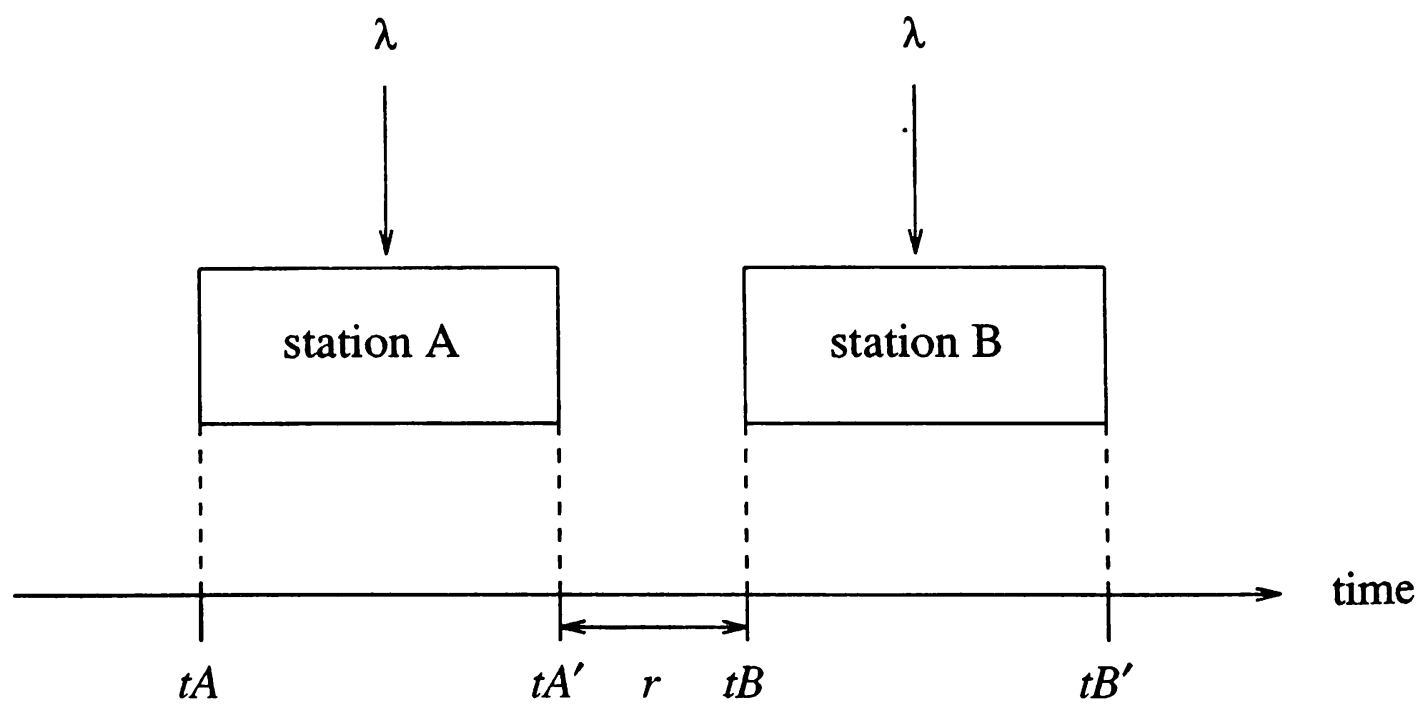

Figure 1. Token ring model 
In the analysis that follows, we first find the state transition probabilities from which we compute the steady state probability. We then derive expressions for average throughput and average packet transmission delay.

\section{STATE TRANSITION PROBABILITY}

\subsection{State Transition Probability from $t A^{\prime}$ to $t B$}

We assume that the states at instant $t A^{\prime}$ and instant $t B$ are represented by $\left(n_{0}, \cdots, n_{7}, f\right)$ and $\left(n_{0}{ }^{\prime}, \cdots, n_{7}, g\right)$, respectively. We also represent the state transition probability from $t A^{\prime}$ to $t B$ by $\mathrm{P}\left(n_{0}^{\prime}, \cdots, n_{7}^{\prime}, g \mid n_{0}, \cdots, n_{7}, f\right)$.

From assumption (5), we find that $\frac{1}{8}\left(1-e^{-\lambda r}\right)$ and $\left(e^{-\lambda r}\right)$ are the probabilities that any station has one or more arrivals of any priority level packet and no arrivals, respectively, between $t A^{\prime}$ and $t B$. By using the multinomial distribution property, we obtain the state transition probability $\mathrm{P}\left(n_{0}{ }^{\prime}, \cdots, n_{7}{ }^{\prime}, g \mid n_{0}, \cdots, n_{7}, f\right)$ as follows:

$$
\mathrm{P}\left(n_{0}, \cdots, n_{7}, g \mid n_{0}, \cdots, n_{7}, f\right)= \begin{cases}\frac{\left[N-\sum_{j=0}^{7} n_{j}\right] !}{\left.\left[\prod_{j=0}^{7}\left[n_{j}^{\prime}-n_{j}\right] !\right] N-\sum_{j=0}^{7} n_{j}^{\prime}\right] !} \\ \cdot\left[\frac{1}{8}\left[1-e^{-\lambda r}\right]\right]_{j=0}^{\sum_{j=0}^{7}\left(n_{j}^{\prime}-n_{j}\right)}\left(e^{-\lambda r]^{N-\sum_{j=0}^{7} n_{j}^{\prime}}} \text { if } f=g\right. \\ 0 & \text { if } f \neq g\end{cases}
$$

where $n_{j} \leq n_{j}{ }^{\prime} \leq N, j=0, \cdots, 7$ and $f, g=0, \cdots, 7$. 


\subsection{State Probability at $t B$}

The probability that station B has a priority level $i$ packet at time instant $t B$ with state $\left(n_{0}, \cdots, n_{7}^{\prime}, f\right)$ is denoted by $\mathrm{P}_{i}\left(n_{0}, \cdots, n_{7}^{\prime}\right)$ and computed as follows:

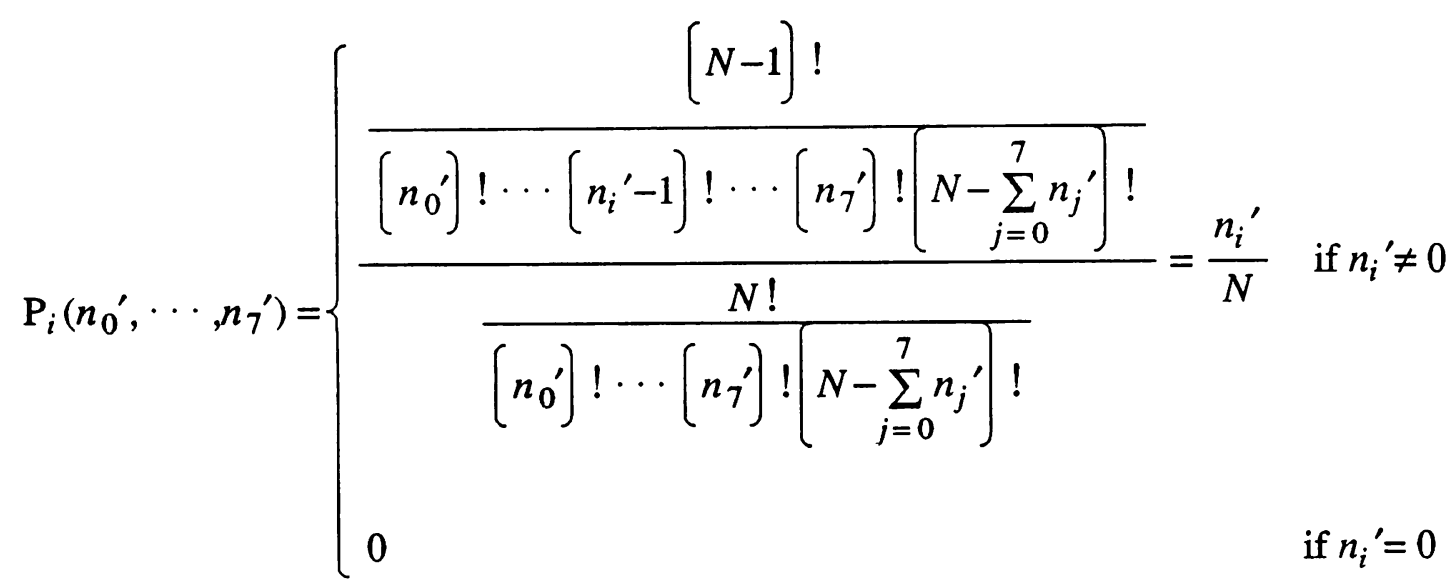

where $0 \leq n_{i}{ }^{\prime} \leq N, i=0, \cdots, 7$.

\subsection{State Transition Probability from $t B$ to $t B^{\prime}$}

The state transition probability from instant $t B$ to instant $t B^{\prime}$ depends on the priority level of a packet in station B and the level of free tokens arrived at and generated from station $B$. If no packet is transmitted, the time duration $t B^{\prime}-t B$ is equal to zero. If a packet is transmitted, then $t B^{\prime}-t B$ is equal to $1+N r$ which is represented by $t$. In the following equations, $X$ denotes the probability that any idle station has a packet arrival before a busy token passes the station.

We consider two cases of the probability. The first case is that both states at $t B$ and $t B^{\prime}$ are the same while the second case is that the states are different. 


\section{Case 1:}

This case occurs when station B has a packet whose priority level is less than the level of the arrived free token or does not have any packet. This case also happens when station B has a packet whose priority level is equal to or greater than the level of the arrived free token. We assume the distances between any two nearest idle stations are the same. The state transition probability of this case is given as follows:

$$
\begin{aligned}
& \mathrm{P}\left(n_{0}^{\prime}, \cdots, n_{7}^{\prime}, i \quad n_{0}^{\prime}, \cdots, n_{7}^{\prime}, i\right)=1-\sum_{j=i}^{7} \mathrm{P}_{j}\left(n_{0}^{\prime}, \cdots, n_{7}^{\prime}\right) \\
& \quad+\mathrm{P}_{i}\left(n_{0}{ }^{\prime}, \cdots, n_{7}{ }^{\prime}\right)\left(\begin{array}{c}
N-\sum_{j=0}^{7} n_{j}^{\prime}+1 \\
1
\end{array}\right]\left[\frac{1}{8}\left[1-e^{-\lambda t}\right]\right]\left[e^{-\lambda t)^{N-\sum_{j=0}^{7} n_{j}^{\prime}} \cdot R(i)}\right. \\
& \quad+P A
\end{aligned}
$$

where

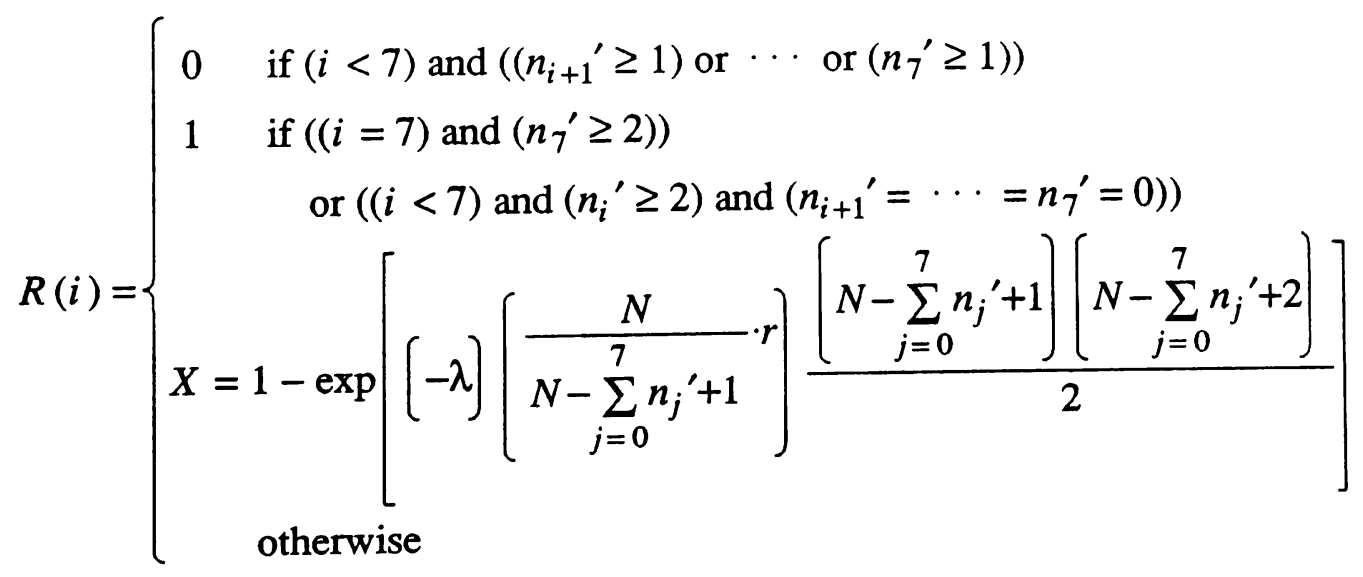




$$
P A=\left\{\begin{array}{c}
\text { if } i=7 \\
\sum_{k=i+1}^{7}\left[\mathrm{P}_{k}\left(n_{0}{ }^{\prime}, \cdots, n_{7}^{\prime}\right)\right. \\
\\
\text { otherwise }
\end{array}\right.
$$

where

$$
R^{\prime}(k)= \begin{cases}0 \quad & \begin{array}{l}
\text { if }\left(n_{i}^{\prime}=0\right) \text { or }\left(n_{i+1}^{\prime} \geq 1\right) \text { or } \cdots \text { or }\left(n_{k-1}{ }^{\prime} \geq 1\right) \text { or }\left(n_{k}{ }^{\prime} \geq 2\right) \\
\text { or }\left(n_{k+1}{ }^{\prime} \geq 1\right) \text { or } \cdots \text { or }\left(n_{7}^{\prime} \geq 1\right) \\
1-X \quad \text { otherwise. }
\end{array}\end{cases}
$$

\section{Case 2:}

This case occurs when station B has a packet whose priority level is equal to or greater than the level of a free token arriving at the station.

$$
\begin{aligned}
& \mathrm{P}\left(n_{0}{ }^{\prime \prime}, \cdots, n_{7}{ }^{\prime \prime}, g \mid n_{0}{ }^{\prime}, \cdots, n_{7}{ }^{\prime}, i\right)=\sum_{k=i}^{7}\left\{\mathrm{P}_{k}\left(n_{0}{ }^{\prime}, \cdots, n_{7}{ }^{\prime}\right)\right. \\
& \left(N-\sum_{j=0}^{7} n_{j}^{\prime}+1\right) ! \\
& \overline{\left[\prod_{j=0}^{7}\left[n_{j}{ }^{\prime \prime}-n_{j}{ }^{\prime}\right) !\right]\left[n_{k}{ }^{\prime \prime}-n_{k}{ }^{\prime}+1\right) !\left(N-\sum_{j=0}^{7} n_{j}^{\prime \prime}\right) !} \\
& \left.\cdot\left[\frac{1}{8}\left[1-e^{-\lambda t}\right]\right]^{\sum_{j=0}^{7}\left(n_{j}^{\prime \prime}-n_{j}{ }^{\prime}\right)+1}\left[e^{-\lambda t}\right]^{N-\sum_{j=0}^{7} n_{j}^{\prime \prime}} \cdot R^{\prime \prime}(k)\right\}
\end{aligned}
$$

where $\left(n_{0}{ }^{\prime \prime}, \cdots, n_{7}{ }^{\prime \prime}, g\right) \neq\left(n_{0}{ }^{\prime}, \cdots, n_{7}{ }^{\prime}, i\right) . R^{\prime \prime}(k)$ has different values depending on the priority levels of a packet and free tokens and is denoted as follows: 
(1) $(g<i \leq k)$ or $(i \leq g<k)$

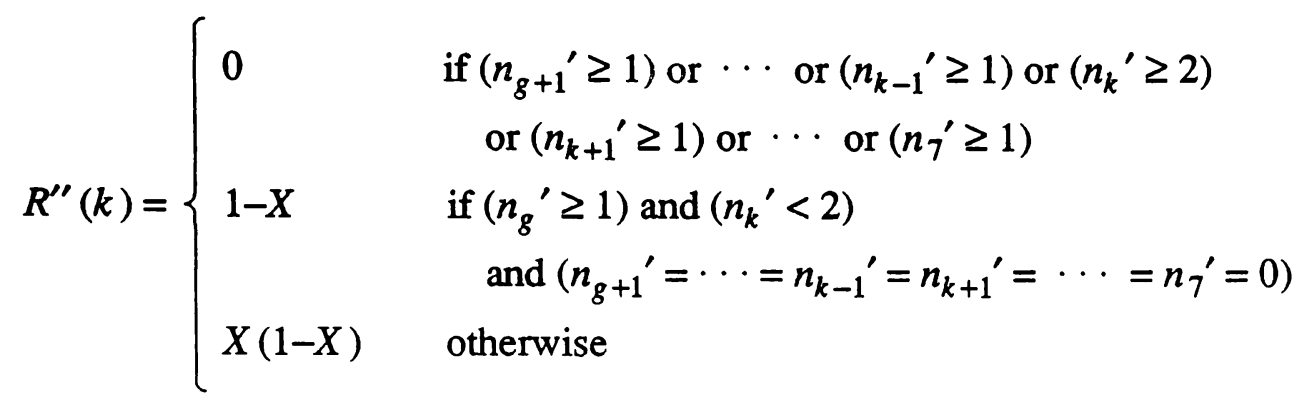

(2) $(i \leq g=k)$

(i) $k<7$

$$
R^{\prime \prime}(k)= \begin{cases}0 & \text { if }\left(n_{g+1}{ }^{\prime} \geq 1\right) \text { or } \cdots \text { or }\left(n_{7}{ }^{\prime} \geq 1\right) \\ 1-X & \text { if }\left(n_{g}{ }^{\prime} \geq 2\right) \text { and }\left(n_{g+1}{ }^{\prime}=\cdots=n_{7}{ }^{\prime}=0\right) \\ X(1-X) & \text { otherwise }\end{cases}
$$

(ii) $k=7$

$$
R^{\prime \prime}(k)= \begin{cases}1 & \text { if }\left(n_{g}{ }^{\prime} \geq 2\right) \\ X & \text { otherwise }\end{cases}
$$

(3) $(i \leq k<g)$

(i) $g<7$

$$
R^{\prime \prime}(k)= \begin{cases}0 & \text { if }\left(n_{g+1} \geq 1\right) \text { or } \cdots \text { or }\left(n_{7}^{\prime} \geq 1\right) \\ 1-X & \text { if }\left(n_{g}^{\prime} \geq 1\right) \text { and }\left(n_{g+1}{ }^{\prime}=\cdots=n_{7}^{\prime}=0\right) \\ X(1-X) & \text { otherwise }\end{cases}
$$

(ii) $g=7$

$$
R^{\prime \prime}(k)= \begin{cases}1 & \text { if }\left(n_{g}{ }^{\prime} \geq 1\right) \\ X & \text { otherwise }\end{cases}
$$




\subsection{Steady State Probability}

Using the state transition probabilities obtained above, we compute the steady state probability $\pi\left(n_{0}{ }^{\prime \prime}, \cdots, n_{7}{ }^{\prime \prime}, g\right)$ as follows:

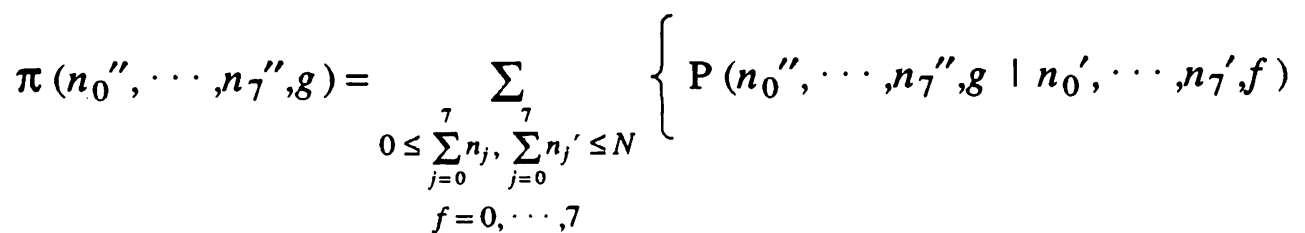

$$
\begin{aligned}
& \left.\cdot \mathrm{P}\left(n_{0}{ }^{\prime}, \cdots, n_{7}{ }^{\prime}, f \mid n_{0}, \cdots, n_{7}, f\right) \cdot \pi\left(n_{0}, \cdots, n_{7}, f\right)\right\}
\end{aligned}
$$

\section{THROUGHPUT AND DELAY ANALYSIS}

\subsection{Average Throughput}

First, we consider the probability $\mathrm{P}\left(k \mid n_{0}, \cdots, n_{7}, f\right)$ that the transmission time, $t B^{\prime}-t B$, is equal to $k$ when the state at time instant $t B$ is $\left(n_{0}, \cdots, n_{7}, f\right)$. If station B has a packet whose priority level is less than the level $f$ of the free token arriving at the station or does not have any packet, the transmission time is 0 . However, if the station has a packet whose priority level is greater than or equal to $f$, the packet is transmitted and the transmission time is equal to $t$. Thus, the probability is represented as follows:

$$
g_{k}=\mathrm{P}\left(k \mid n_{0}, \cdots, n_{7}, f\right)= \begin{cases}1-\sum_{j=f}^{7} \mathrm{P}_{j}\left(n_{0}, \cdots, n_{7}\right) & \text { if } k=0 \\ \sum_{j=f}^{7} \mathrm{P}_{j}\left(n_{0}, \cdots, n_{7}\right) & \text { if } k=t \\ 0 & \text { otherwise }\end{cases}
$$


In addition, the probability generating function of the probability $g_{k}$ is represented by $\mathrm{G}\left(z \mid n_{0}, \cdots, n_{7}, f\right)$ and computed as follows:

$$
\begin{aligned}
\mathrm{G}\left(z \mid n_{0}, \cdots, n_{7}, f\right) & =\sum_{k} z^{k} g_{k}=z^{0} g_{0}+z^{t} g_{t} \\
& =1-\sum_{j=f}^{7} \mathrm{P}_{j}\left(n_{0}, \cdots, n_{7}\right)+z^{t} \cdot \sum_{j=f}^{7} \mathrm{P}_{j}\left(n_{0}, \cdots, n_{7}\right) \\
& =1+\left(z^{t}-1\right) \cdot \sum_{j=f}^{7} \mathrm{P}_{j}\left(n_{0}, \cdots, n_{7}\right)
\end{aligned}
$$

Then, the first derivative of $\mathrm{G}\left(z \mid n_{0}, \cdots, n_{7}, f\right)$ evaluated at $z=1$ yields the average transmission time $\mathrm{F}\left(n_{0}, \cdots, n_{7}, f\right)$ and is given by

$$
\begin{aligned}
\mathrm{F}\left(n_{0}, \cdots, n_{7}, f\right) & \left.=\mathrm{G}^{(1)}\left(1 \mid n_{0}, \cdots, n_{7}, f\right)=\frac{d \mathrm{G}\left(z \mid n_{0}, \cdots, n_{7}, f\right)}{d z}\right]_{z=1} \\
& =t \cdot \sum_{j=f}^{7} \mathrm{P}_{j}\left(n_{0}, \cdots, n_{7}\right)=\frac{t \cdot \sum_{j=f}^{7} n_{j}}{N}
\end{aligned}
$$

From the above equations, we now derive the average throughput of any priority level packet. In the following equation, $\mathrm{T}_{i}$ denotes the average throughput of level $i$ packet.

$$
\begin{aligned}
& \sum_{0 \leq \sum_{j=0}^{7} n_{j} \leq N} \pi\left(n_{0}, \cdots, n_{7}, f\right) \cdot \mathrm{PR}_{i}\left(n_{0}, \cdots, n_{7}, f\right) \\
& \mathrm{T}_{i}=\frac{f=0, \cdots, 7}{\sum_{7} \pi\left(n_{0}, \cdots, n_{7}, f\right) \cdot \mathrm{F}\left(n_{0}, \cdots, n_{7}, f\right)} \quad i=0, \cdots, 7 \\
& 0 \leq \sum_{j=0} n_{j} \leq N \\
& f=0, \cdots, 7
\end{aligned}
$$


where $\mathrm{PR}_{i}\left(n_{0}, \cdots, n_{7}, f\right)$ is the probability that a priority level $i$ packet is transmitted at state $\left(n_{0}, \cdots, n_{7}, f\right)$. Since a packet is transmitted when its priority level $i$ is equal to or greater than the priority level $f$ of a free token, we have the following equation for $\mathrm{PR}_{i}\left(n_{0}, \cdots, n_{7}, f\right)$ :

$$
\mathrm{PR}_{i}\left(n_{0}, \cdots, n_{7}, f\right)= \begin{cases}0 & \text { if } i<f \\ \mathrm{P}_{i}\left(n_{0}, \cdots, n_{7}\right) & \text { otherwise }\end{cases}
$$

\subsection{Average Transmission Delay}

To derive the average transmission delay, we first consider the probability $\mathrm{PN}_{i}\left(m_{i} \mid n_{0}, \cdots, n_{7}, f, k\right)$ where $m_{i}$ is the number of stations which have level $i$ packet at the time instant that $k$ time units have elapsed after a free token was issued in the state $\left(n_{0}, \cdots, n_{7}, f\right)$. The probability is computed as follows:

$$
\mathrm{PN}_{i}\left(m_{i} \mid n_{0}, \cdots, n_{7}, f, k\right)=\left[\begin{array}{c}
N-\sum_{j=0}^{7} n_{j} \\
m_{i}-n_{i}
\end{array}\right] \cdot\left[\frac{1}{8}\left[1-e^{-\lambda k}\right]\right]^{m_{i}-n_{i}} \cdot\left(e^{-\lambda k}\right)^{N-\sum_{j=0}^{7} n_{j}-m_{i}+n_{i}}
$$

where $n_{i} \leq m_{i} \leq N-\sum_{j=0}^{7}+n_{i}$ and $i=0, \cdots, 7$.

Next, we calculate the average number of level $i$ packets in the network at the instant that $k$ time units have elapsed after a free token was issued in the state $\left(n_{0}, \cdots, n_{7}, f\right)$. This number is denoted by $\mathrm{N}_{i}\left(n_{0}, \cdots, n_{7}, f, k\right)$ and given by 


$$
\begin{aligned}
& \mathrm{N}_{i}\left(n_{0}, \cdots, n_{7}, f, k\right)=\sum_{m_{i}=n_{i}}^{n_{i}+N-\sum_{j=0}^{7} n_{j}} m_{i} \cdot \mathrm{PN}_{i}\left(m_{i} \mid n_{0}, \cdots, n_{7} f, k\right) \\
& =\left[\frac{1}{8}\left(1+7 e^{-\lambda k}\right]\right]^{N-\sum_{j=0}^{7} n_{j}-1} \cdot\left[\frac{1}{8}\left[1-e^{-\lambda k}\right] \cdot\left[N-\sum_{j=0}^{7} n_{j}\right]+\frac{n_{i}}{8}\left[1+7 e^{-\lambda k}\right]\right]
\end{aligned}
$$

In addition, we consider the average number of level $i$ packets in the network from the generation of a free token in state $\left(n_{0}, \cdots, n_{7}, f\right)$ to the next generation of a free token. This number is given as follows:

$$
\mathrm{M}_{i}\left(n_{0}, \cdots, n_{7}, f\right)=\mathrm{N}_{i}\left(n_{0}, \cdots, n_{7}, f, t+r\right) \cdot \mathrm{P}\left(t \mid n_{0}, \cdots, n_{7}, f\right)
$$

From the above equations, we compute the average number of level $i$ packets in the network at any arbitrary time and this is given by

$$
\begin{aligned}
\sum_{\substack{7 \\
0 \leq \sum_{j=0}^{7} n_{j} \leq N}} \pi\left(n_{0}, \cdots, n_{7}, f\right) \cdot \mathrm{M}_{i}\left(n_{0}, \cdots, n_{7}, f\right) \\
\mathrm{L}_{i}=\frac{f=0, \cdots, 7}{\sum_{\substack{7 \\
j=0}} \pi\left(n_{0}, \cdots, n_{7}, f\right) \cdot \mathrm{F}\left(n_{0}, \cdots, n_{7}, f\right)} \quad i=0, \cdots, 7 \\
f=0, \cdots, 7
\end{aligned}
$$

We now know the average throughput and the average number of any priority level packets. Therefore, we can use Little's law to calculate the average transmission delay $\mathrm{D}_{i}$ as follows:

$$
\mathrm{D}_{i}=\frac{\mathrm{L}_{i}}{\mathrm{~T}_{i}}
$$




\section{PERFORMANCE MEASUREMENTS}

In order to validate results obtained using the above analytic model, the results are compared with results from a simulation model. For simplicity of analysis, we assume there are two levels of priority, 0 and 1 , where 1 is higner level. When different values are assigned to a certain parameter, the other parameters have the following values:

Transmission rate : $4 \mathrm{Mbits} / \mathrm{sec}$

Number of stations $(N): 10$

Delay between two stations $(r): 2$ micro-seconds

Packet inter-arrival time $(\lambda): 0.05$ seconds

Packet length : 1000 bytes

Figure 2 graphically shows the average transmission delay as a function of packet inter-arrival time,and Figure 3 shows the delay as a function of packet length. In both figures, solid and dashed lines indicate analytic and simulation results, respectively. 


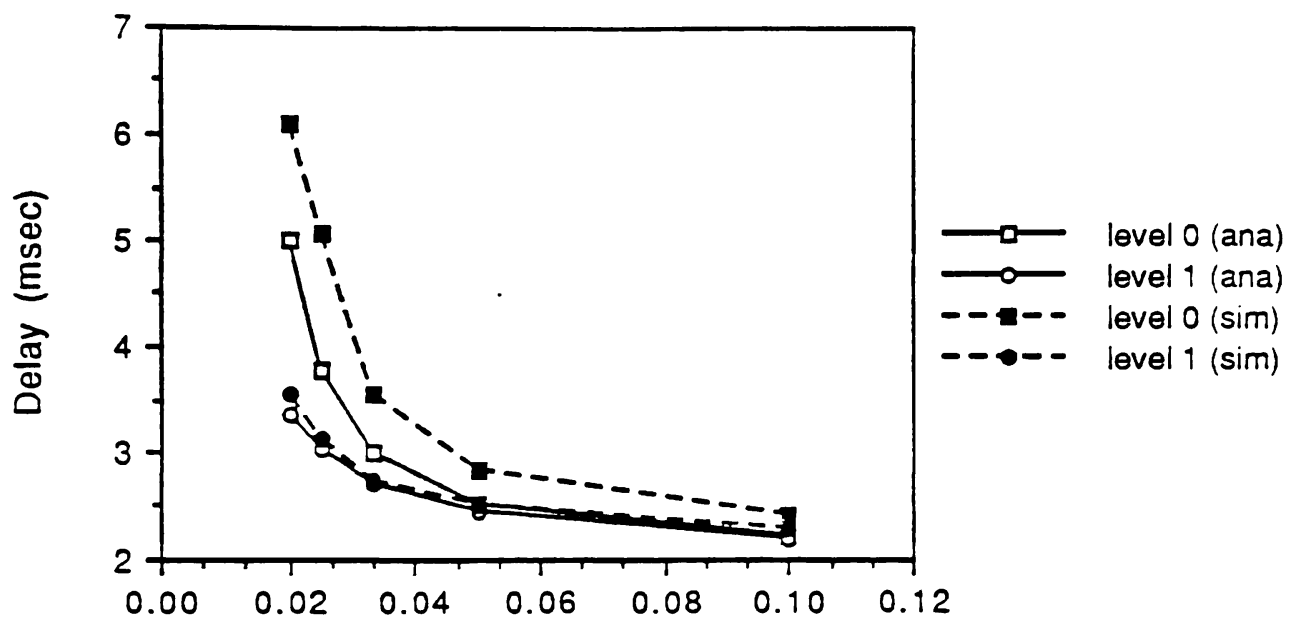

Inter-arrival time (sec)

Figure 2. Transmission delay vs. Inter-arrival time

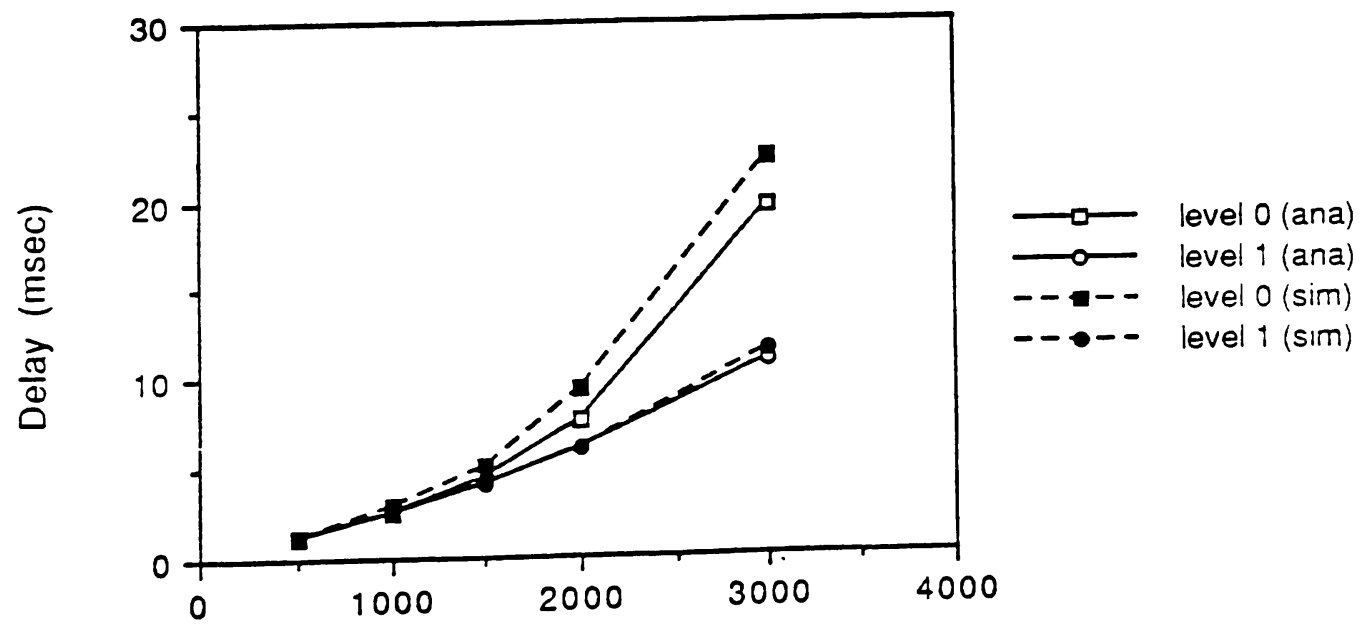

Packet Length (bytes)

Figure 3. Transmission delay vs. Packet length 


\section{CONCLUSIONS}

In this report, we have introduced a prioritized token ring protocol and an analytic model for evaluating its performance. The average throughput and transmission delay are obtained by means of a Markov chain model. In addition, average packet transmission delay is graphically shown as functions of inter-arrival time and packet length.

In order to verify our analytic model, the results are compared with the results obtained from simulation. From the comparison, we have observed that analytic and simulation results are consistent with each other for high priority level packet. However, there is a small discrepancy for low priority level packet but this discrepancy is acceptable. 


\section{REFERENCES}

[1] Z. Shen, S. Masuyama, S. Muro, T. Hasegawa, "Performance evaluation of prioritized token ring protocols," Eleventh International Teletraffic Congress, M. Akiyama (ed.), pp. 4.2A-3-1 - 4.2A-3-7, Elsevier Science Publishers B.V. (NorthHolland), Sept. 1985

[2] IEEE Standard 802.5, "Local area networks: Token Ring access method and physical layer specifications," 1985 